\title{
openheart Contemporary trends in use of mechanical circulatory support in patients with acute MI and cardiogenic shock
}

\author{
Ole Kristian Lerche Helgestad (D) , , ${ }^{1,2,3}$ Jakob Josiassen, ${ }^{4}$ Christian Hassager, ${ }^{4,5}$ \\ Lisette Okkels Jensen, ${ }^{1}$ Lene Holmvang, ${ }^{4,5}$ Nanna Louise Junker Udesen, ${ }^{1}$ \\ Henrik Schmidt, ${ }^{6}$ Hanne Berg Ravn, ${ }^{5,7}$ Jacob Eifer Moller ${ }^{1,3}$
}

To cite: Helgestad OKL, Josiassen J, Hassager C, et al. Contemporary trends in use of mechanical circulatory support in patients with acute $\mathrm{Ml}$ and cardiogenic shock. Open Heart 2020;7:e001214. doi:10.1136/ openhrt-2019-001214

Received 8 December 2019 Revised 15 January 2020 Accepted 6 February 2020
Check for updates

(C) Author(s) (or their employer(s)) 2020. Re-use permitted under CC BY-NC. No commercial re-use. See rights and permissions. Published by BMJ.

For numbered affiliations see end of article.

Correspondence to Dr Ole Kristian Lerche Helgestad; Ole.MollerHelgestad@rsyd.dk

\section{ABSTRACT}

Objectives To describe the contemporary trends in the use of mechanical circulatory support (MCS) in patients with acute myocardial infarction and cardiogenic shock (AMICS). To evaluate survival benefit with early application of intra-aortic balloon pump (IABP) or Impella CP.

Methods A cohort study of all consecutive patients with AMICS undergoing percutaneous coronary intervention (PCl) $<24$ hours of symptom onset (early PCl) in southeastern Denmark from 2010 to 2017. A matched case-control study comparing 30-day mortality between patients receiving early-IABP or early-Impella CP and their respective control group. Controls were matched on age, left ventricular ejection fraction, arterial lactate, estimated glomerular filtration rate and cardiac arrest before $\mathrm{PCl}$. Early-IABP/Impella CP was defined as applied before PCI if shock developed pre-PCl, or immediately after $\mathrm{PCl}$ if shock developed during $\mathrm{PCl}$.

Results 903 patients with AMICS undergoing early PCI were identified. Use of MCS decreased from $50 \%$ in 2010 to $25 \%$ in 2017, p for trend of $<0.001$. The IABP was abandoned in 2012 and replaced mostly by Impella CP. Patients receiving MCS in 2013-2017 had more compromised haemodynamics compared with patients receiving MCS in 2010-2012. 40 patients received early IABP, and 40 patients received early Impella CP. Only the group receiving early Impella CP was associated with lower 30-day mortality compared with their matched control group (30-day mortality $40 \%$ vs $77.5 \%$, plog-rank of $<0.001$ ).

Conclusion Use of MCS decreased by $50 \%$ from 2010 to 2017. Patients receiving MCS had more compromised haemodynamics in recent years. Early application of Impella CP was associated with reduced 30-day mortality compared with a matched control group.

\section{INTRODUCTION}

Cardiogenic shock remains a leading cause of death following acute myocardial infarction with sustained 30-day mortality of approximately $50 \%$ for nearly two decades. ${ }^{12}$ Use of mechanical circulatory support (MCS) is widely debated with no substantial evidence

\section{Key questions}

What is already known about this subject?

- There is no randomised controlled trial to support the use of mechanical circulatory support (MCS) in patients with acute myocardial infarction and cardiogenic shock. Little is known about the patient population receiving MCS in recent years. Observational studies show conflicting results but generally support implementation of MCS before revascularisation.

What does this study add?

- The intra-aortic balloon pump (IABP) was replaced by Impella CP and, to a lesser extent, venoarterial membrane oxygenation following the neutral IABPSHOCK II trial. Fewer patients received MCS after IABP-SHOCK II, and patients receiving MCS in recent years had more compromised haemodynamics at the time of shock compared with earlier years. When taking the timing of shock into consideration, not only prepercutaneous coronary intervention (PCI) implementation but also immediate post-PCI implementation of Impella CP was associated with improved outcome compared with a matched control group.

How might this impact on clinical practice?

- This study does not support general use of MCS but if MCS is required, this observational study supports early use of Impella CP defined as pre-PCl, or immediately after $\mathrm{PCl}$ if shock develops during the procedure.

supporting its use in patients with acute myocardial infarction and cardiogenic shock (AMICS). To date, the Intra-aortic Balloon Pump (IABP)-SHOCK II trial is the only adequately powered randomised controlled trial assessing the use of MCS in AMICS, and the trial failed to show any prognostic benefit with the use of IABP. $^{3}$ Contemporary studies on temporal trends in use of MCS in AMICS are limited but generally show a 


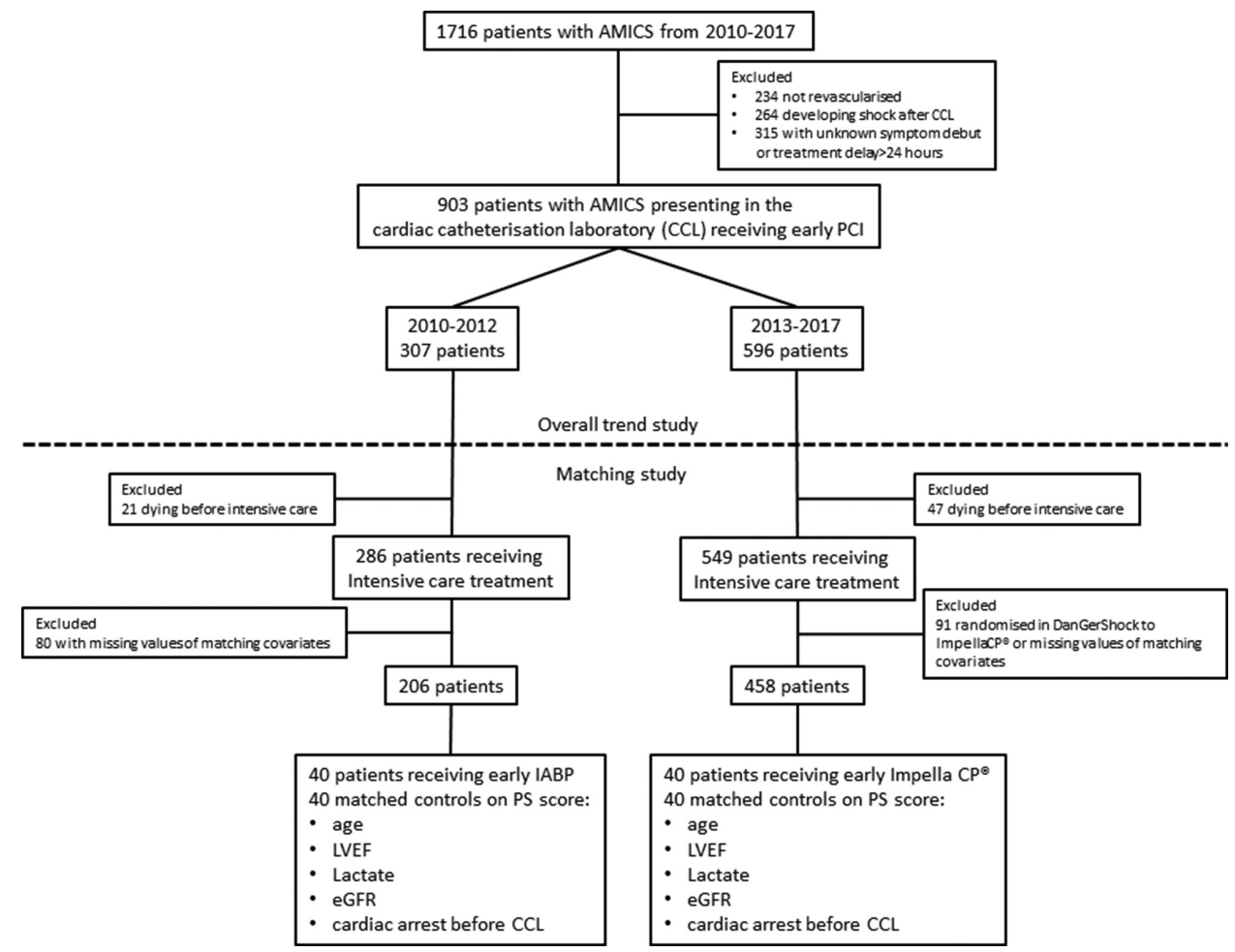

Figure 1 Consort diagram of patient selection. Dotted line indicates the cut-off between the trend study and the matching study. AMICS, acute myocardial infarction and cardiogenic shock; CCL, cardiac catheterisation laboratory; eGFR, estimated glomerular filtration rate; IABP, intra-aortic balloon pump; LVEF, left ventricular ejection fraction; PCI, percutaneous coronary intervention.

shift in choice of the device with a decrease in the use of IABP and an increase in the use of other devices, namely, venoarterial extracorporeal membrane oxygenation (VAECMO) and transvalvular microaxial flow pumps. ${ }^{45} \mathrm{In}$ a recent retrospective study, general use of Impella was not associated with improved survival compared with the IABP. ${ }^{6}$ However, the percutaneous assist devices pooled in the analyses have differing support capabilities and mode of action, and there is increasing evidence of a possible timing issue favouring early application of MCS . ${ }^{7-12}$ Therefore, we sought to investigate the use of MCS in AMICS and assessed the temporal trends in the choice of the device and the patient characteristics of those receiving MCS between 2010 and 2017. Further, we assessed the survival benefit of early application of IABP or Impella CP compared with a matched control group.

\section{METHODS}

\section{Study population}

All patients fulfilling the criteria for AMICS in the cardiac catheterisation laboratory undergoing early percutaneous coronary intervention (PCI) (within 24hours of symptom onset) from 2010 to 2017 were identified in the RETROSHOCK registry (figure 1). RETROSHOCK is a retrospective registry including all consecutive patients with an individually validated diagnosis of AMICS, admitted to two tertiary cardiac centres that provide AMICS care for two-thirds of the Danish population. ${ }^{1}$ AMICS was defined as persistent hypotension (systolic blood pressure of $\leq 90 \mathrm{~mm} \mathrm{Hg}$ for $>30 \mathrm{~min}$ or use of vasoactive drugs), end-organ hypoperfusion (altered mental status, cold/clammy skin, oliguria or arterial lactate $\geq 2.5 \mathrm{mmol} / \mathrm{L}$ ) and reduced cardiac function, caused by an acute myocardial infarction.

\section{Intervention and data collection}

The intervention of interest was the use of short-term MCS and the device type (IABP, VA-ECMO, Impella V.2.5, Impella CP, Impella RP and Impella V.5.0) from 2010 to 2017. Patient characteristics were obtained at the time of shock (ie, in the cardiac catheterisation laboratory). Patients were stratified as those who received MCS versus those who did not (MCS vs no MCS), in addition to the time period (2010-2012 vs 2013-2017). Mean arterial pressure was calculated as diastolic blood pressure $+0.33 \times$ pulse pressure. Estimated glomerular filtration rate (eGFR) was calculated based on age, gender and creatinine on arrival according to the Chronic Kidney Disease Epidemiology Collaboration equation. ${ }^{13}$ Early MCS application was defined as the initiation of IABP/Impella CP before PCI in patients with shock prior to the procedure or before leaving the cardiac catheterisation laboratory in patients developing shock during the procedure. Timing of shock was determined as pre-PCI versus during PCI based on blood pressure, if not specified in the medical record. Additional MCS was defined as the use of more than one device. In the matched study, MCS escalation was defined as the transition to a more 
powerful device (ie, from no device to any MCS, IABP to any other MCS, Impella CP to VA-ECMO and Impella CP to Impella V.5.0). Patient delay was defined as the time from symptom onset to first medical contact. System delay was defined as the time from first medical contact to insertion of the guiding catheter.

\section{Study endpoints}

The primary endpoint of this study was 30-day all-cause mortality in the intervention group and the matched control group. Secondary endpoints were the mode of in-hospital death (cardiac, anoxic brain damage or multiorgan failure) for the matched cohort and 30-day mortality for the entire cohort.

\section{Propensity matching}

Use of MCS was considered equal to initiating intensive care treatment, along with intubation and vasoactive drug infusion. Patients who died in the catheterisation laboratory before receiving any form of intensive care treatment were considered not to be potential candidates for MCS and therefore were excluded from the propensity matched analysis (figure 1). Among the patients from 2010 to 2012, those receiving an early IABP were matched 1:1 to their nearest neighbour according to a propensity score. Likewise, among the patients from 2013 to 2017, those receiving an early Impella CP were matched 1:1 to their nearest neighbour according to a propensity score (figure 1). The following baseline characteristics were used for calculating the propensity score as they have been identified as significant risk factors for death in AMICS: age (5-year increments), left ventricular ejection fraction (LVEF, increments of 5\%), arterial lactate at the time of shock $(\leq 2.0,2.1-4.0,4.1-6.0,6.1-8.0,8.1-10.0,10.1-$ 12.0 and $>12.0 \mathrm{mmol} / \mathrm{L}$ ), eGFR (grouped as $<30,30-44$, $45-59,60-89$ and $\geq 90 \mathrm{~mL} / \mathrm{min} / 1.73 \mathrm{~m}^{2}$ ) and resuscitation for cardiac arrest before arrival in the cardiac catheterisation laboratory. We did not identify any residual confounding of age, eGFR or lactate as continuous variables within each respective stratum. Patients randomised to Impella CP as part of the ongoing DanGer Shock trial (NCT01633502) were excluded from the matched study (figure 1). ${ }^{14}$

\section{Statistics}

Patient characteristics among those receiving MCS and those not receiving MCS were compared both within the same period and across different periods (MCS 20102012 vs MCS 2013-2017, no MCS 2010-2012 vs no MCS 2013-2017, MCS 2010-2012 vs no MCS 2010-2012 and MCS 2013-2017 vs no MCS 2013-2017). Student's t-test was used to compare Gaussian data reported as mean (SD); Wilcoxon rank-sum test was used to compare nonGaussian data reported as median $(\mathrm{Q} 1, \mathrm{Q} 3)$, and $\chi^{2}$ test (Fisher's exact test for sparse data) was used to compare proportions reported as number (percentage). Thirty-day mortality was estimated using the Kaplan-Meier method, and differences between groups were compared using

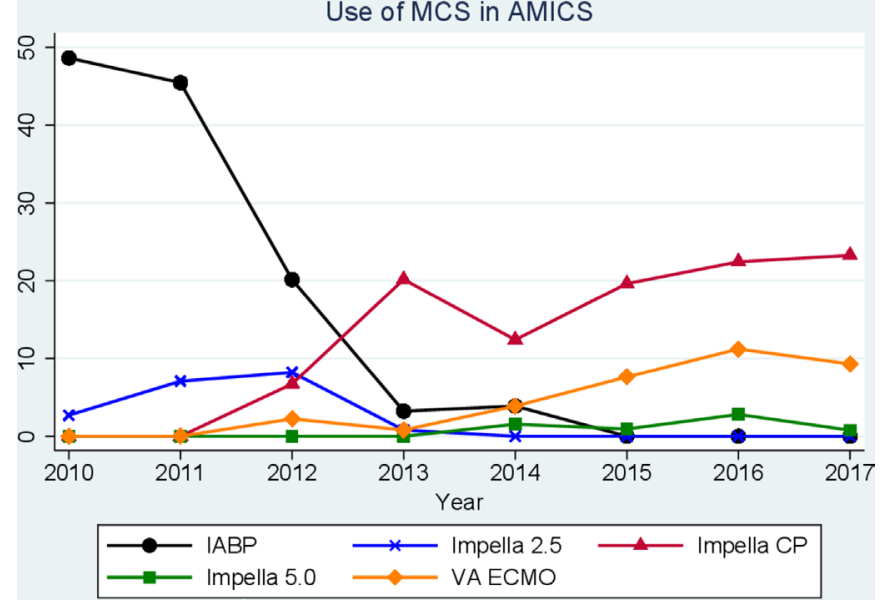

Figure 2 Temporal trends in the use of MCS from 2010 to 2017 with different Impella devices delineated. AMICS, acute myocardial infarction and cardiogenic shock; IABP: intra-aortic balloon pump; MCS, mechanical circulatory support; VA-ECMO: venoarterial extracorporeal membrane oxygenation.

the log-rank test. All statistical analyses were performed using STATA software V.14.2.

\section{RESULTS}

A total of 903 patients presenting to the cardiac catheterisation laboratory with AMICS undergoing early PCI between 2010 and 2017 were identified in the registry (figure 1). The use of MCS decreased from 50\% in 2010 to $25 \%$ in 2017 (figure 2). There was a major shift in the choice of MCS since 2012 with a substantial decrease in the application of IABP and a concomitant increase in the application of Impella CP (figure 2). Given the marked change in the choice of device use over time, we compared patients receiving MCS before 2012 with those receiving MCS after 2012. Patients receiving MCS after 2012 were younger and had more compromised haemodynamics at the time of shock compared with patients receiving MCS before 2012 (table 1 and figure 3). Among patients not receiving MCS, more comorbidity and slightly more compromised haemodynamics were observed in those treated after 2012 compared with those treated before 2012. Otherwise, patient characteristics were balanced across the two eras (table 1). Patients not receiving MCS had nearly identical 30-day mortality $(48 \%, \mathrm{p}=0.95)$ across eras, but the need for transfusion was more frequent in the period before 2012.

We identified 40 patients receiving early IABP and 40 patients receiving early Impella CP. As all patients receiving early IABP/Impella CP survived to intensive care treatment, the matched controls were also limited to patients receiving intensive care. Values of matching covariates and other patient characteristics can be found in table 2. The Impella CP group had significantly lower 30-day mortality compared with the matched control group, corresponding to an average number needed to treat of 2.7 (30-day mortality $40.0 \%$ with early-Impella 


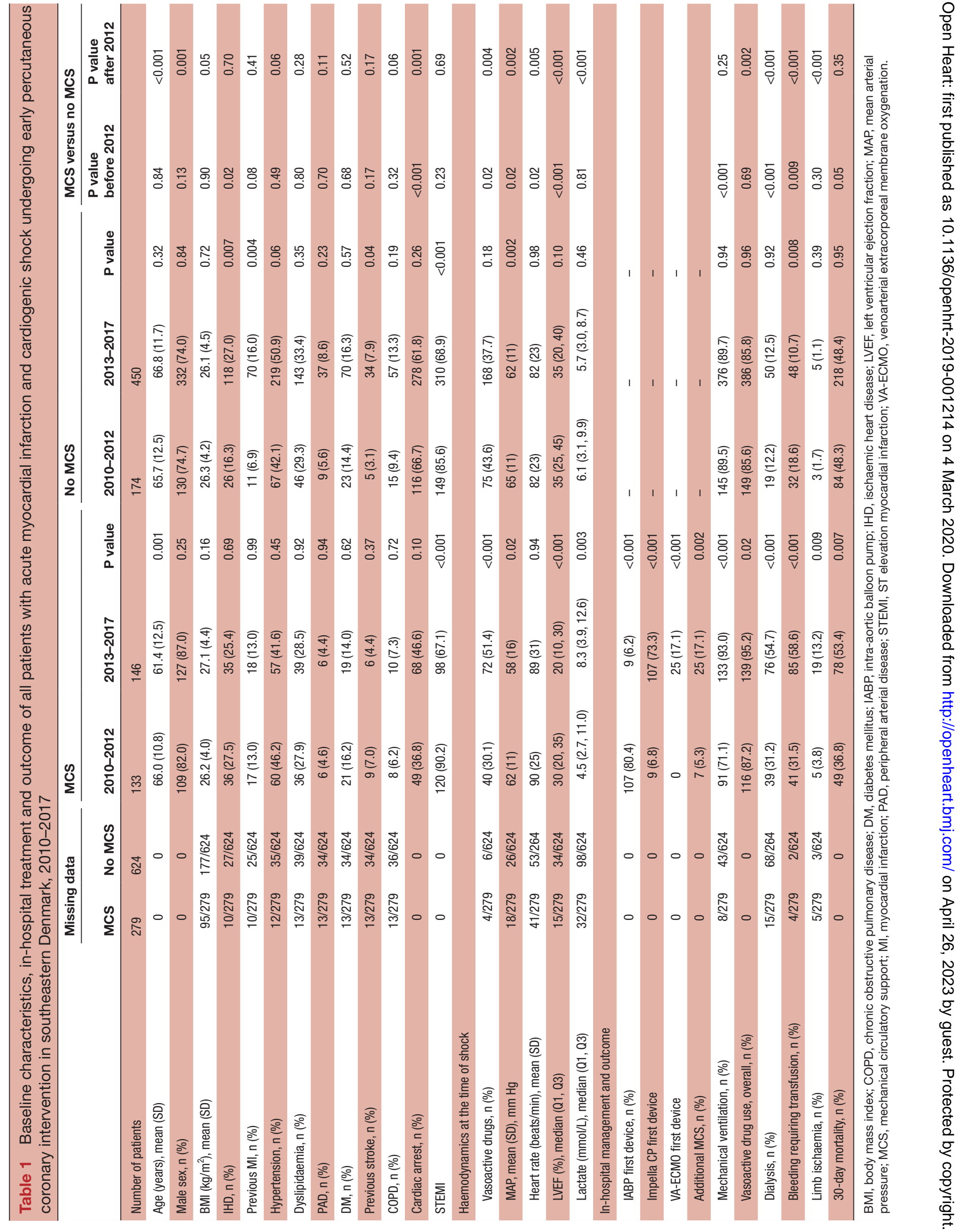



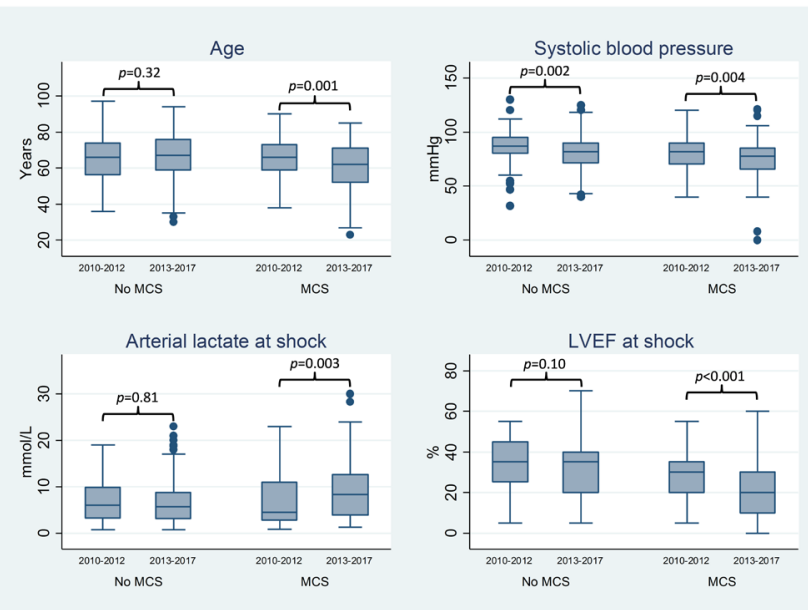

Figure 3 Box plot for age, systemic systolic blood pressure, arterial lactate and LVEF in patients receiving MCS versus not receiving MCS in the pre-IABP-SHOCK II (2010-2012) and post-IABP-SHOCK II period (2013-2017). IABP, intraaortic balloon pump; LVEF, Left ventricular ejection fraction; MCS, mechanical circulatory support.

CP vs $77.5 \%$ with control, $\mathrm{p}<0.001$; table 2 and figure 4 ). The improved outcome in the early Impella CP group was mainly driven by fewer in-hospital cardiac deaths. Although there were numerically fewer deaths in the early IABP group compared with the matched control group, no statistically significant difference was observed (30-day mortality $27.5 \%$ with early IABP vs $37.5 \%$ with control, $\mathrm{p}=0.35$ ). Following exclusion of patients developing shock during the procedure, 30-day mortality was for early Impella CP versus control: $40.9 \%$ vs $79.4 \%$, $\mathrm{p}=0.005$, and for early-IABP versus control: $36.0 \%$ vs $41.2 \%, \mathrm{p}=0.61$.

\section{DISCUSSION}

The current study demonstrates major changes in the use of short-term MCS over the last decade. Patients receiving MCS in recent years were younger and had more profound cardiogenic shock compared with patients receiving MCS in previous years. Interestingly, the choice of MCS device changed drastically from IABP to mostly Impella CP after the IABP-SHOCK II trial in 2012. Early application of Impella CP was associated with lower 30-day mortality compared with the matched controls, a trend that was not be observed among patients with early application of IABP.

Previous studies from Europe and the USA have demonstrated similar temporal trends regarding the choice of MCS system with decreasing use of IABP and increasing use of VA-ECMO, axial pump-related devices and Tandem Heart, but not to the same degree as observed in the present study. ${ }^{41516}$ The abandonment of IABP coincides with the published results of the IABP-SHOCK II trial, and a similar change in practice has been reported from a single-centre in Berlin. ${ }^{17}$ The present study is the first study to delineate between the Impella family of devices and to highlight the increased application of
Impella CP for AMICS in recent years. It should be noted that Impella V.2.5 was used in the early years; however, its use decreased concomitant with the abandonment of IABP following the introduction of Impella CP. Also, the initiation of the randomised DanGer Shock trial (NCT01633502) involving the Impella CP in 2013 and patient recruitment at the two participating institutions likely led to increased awareness of Impella CP. ${ }^{14}$ In addition, clinicians might have favoured the use of Impella CP over Impella V.2.5 given the increased blood flow by the Impella CP. Studies reporting on contemporary trends on patient populations receiving MCS is scarce, and this study shows that patients receiving MCS were younger and more haemodynamically compromised in recent years (2013-2017) compared with earlier years (2010-2012). MCS was applied in $\sim 25 \%$ of cases in the post-IABP-SHOCK II era compared with $\sim 50 \%$ in the pre-IABP-SHOCK II era, and was probably restricted to patients with more profound shock, explaining the more compromised haemodynamics of patients receiving MCS in recent years. This concept is further supported by the observed increase in mechanical ventilation, vasoactive drugs, dialysis and higher 30-day mortality in the MCS group from the post-IABP-SHOCK II era versus preIABP-SHOCK II era. Given the striking differences in the patient characteristics of those receiving MCS before and after 2012, we exclusively compared the treatment effects of early IABP/Impella CP in the two separate time eras.

In agreement with the IABP-SHOCK II trial, early application of IABP compared with the matched control group was not associated with improved outcome in this study. ${ }^{3}$ Early application of Impella CP was associated with improved 30-day mortality corresponding to an average number needed to treat of three to avoid one fatality, assuming that the intervention drove the difference. This contrasts with the recent IMPRESS (MPella versus IABP Reduces mortality in STEMI patients treated with primary PCI in Severe cardiogenic SHOCK) randomised trial of 48 patients, which did not find an overall survival benefit of Impella CP compared with IABP. ${ }^{10}$ In the trial, 91.7\% of patients had cardiac arrest before randomisation and might represent an entirely different clinical entity compared with the mixed cohort of patients with AMICS. The trial did, however, report an absolute risk reduction of $28 \%$ in 30-day mortality when MCS was applied pre-PCI, although the numbers were small and difference did not reach statistical significance. ${ }^{10}$ A recent single-centre observational study found a non-significant absolute $15 \%$ lower 30-day mortality among 62 patients receiving an Impella compared with 54 patients receiving an IABP, despite the fact that patients receiving Impella had more often received out-of-hospital cardiopulmonary resuscitation, had lower LVEF and had higher lactate levels at device implementation. ${ }^{17} \mathrm{~A}$ recent multicentre observational study found no survival benefit with general use of Impella V.2.5/CP compared with matched patients from the IABPSHOCK II trial cohort, but did report a non-significant absolute $10.6 \%$ lower 30 -day mortality for Impella in the 
Table 2 Baseline characteristics, in-hospital treatment and outcome for the matched cohort

\begin{tabular}{|c|c|c|c|c|c|c|}
\hline & Early IABP v & us control & & Early Impella CP & ersus control & \\
\hline & Early IABP & Control & $P$ value & Early ImpellaCP & Control & $P$ value \\
\hline Number of patients & 40 & 40 & & 40 & 40 & \\
\hline Age (years), mean (SD) & $68.8(11.1)$ & $69.1(9.9)$ & 0.90 & $64.5(10.1)$ & $65.9(13.3)$ & 0.60 \\
\hline Male sex, n (\%) & $33(82.5)$ & $28(70.0)$ & 0.19 & $34(85.5)$ & $29(72.5)$ & 0.17 \\
\hline BMI $\left(\mathrm{kg} / \mathrm{m}^{2}\right)$, mean $(\mathrm{SD})$ & $25.5(3.7)$ & $26.6(4.0)$ & 0.28 & 27.3 & 24.9 & 0.01 \\
\hline IHD, n (\%) & $11(27.5)$ & $8(20.5)$ & 0.47 & $14(36.8)$ & $17(43.6)$ & 0.55 \\
\hline Previous Ml, n (\%) & $3(7.5)$ & $4(10.3)$ & 0.71 & $8(21.1)$ & $8(20.0)$ & 0.91 \\
\hline Hypertension, n (\%) & $25(64.1)$ & $15(39.5)$ & 0.03 & $22(59.5)$ & $23(59.0)$ & 0.97 \\
\hline Dyslipidaemia, n (\%) & $11(28.2)$ & $9(23.7)$ & 0.65 & $15(40.5)$ & $16(41.0)$ & 0.97 \\
\hline PAD, n (\%) & $1(2.5)$ & $2(5.1)$ & 0.61 & $2(5.4)$ & $6(15.8)$ & 0.26 \\
\hline DM, n (\%) & $9(22.5)$ & $6(15.4)$ & 0.42 & $7(18.9)$ & $4(10.5)$ & 0.35 \\
\hline Previous stroke, $\mathrm{n}(\%)$ & $4(10.3)$ & $2(5.1)$ & 0.68 & $1(2.7)$ & $1(2.6)$ & 1.00 \\
\hline COPD, n (\%) & $1(2.6)$ & $3(7.7)$ & 0.62 & $0(0.0)$ & $2(5.3)$ & 0.49 \\
\hline Cardiac arrest, $\mathrm{n}(\%)$ & $9(22.5)$ & $10(25.0)$ & 0.79 & $16(40.0)$ & $15(37.5)$ & 0.82 \\
\hline Time to ROSC (min), median (Q1, Q3) & $25(18,25)$ & $27(17,33)$ & 0.71 & $23(15,75)$ & $27.5(15,70)$ & 0.61 \\
\hline STEMI, $n(\%)$ & $36(90.0)$ & $38(95.0)$ & 0.40 & $27(67.5)$ & $24(60.0)$ & 0.49 \\
\hline Patient delay (min), median (Q1, Q3) & $75(20,256)$ & $108(24,205)$ & 0.91 & $34(2,229)$ & $46(14,219)$ & 0.46 \\
\hline System delay (min), median $(Q 1, Q 3)$ & $149(115,194)$ & $164(120,245)$ & 0.41 & $140(105,175)$ & $142(100,250)$ & 0.75 \\
\hline Haemodynamics at the time of shock & & & & & & \\
\hline Shock before $\mathrm{PCl}$ procedure, n (\%) & $25(62.5)$ & $34(85.0)$ & 0.02 & $22(55.0)$ & $34(85.0)$ & 0.003 \\
\hline Vasoactive drugs, $\mathrm{n}(\%)$ & $27(67.5)$ & $27(67.5)$ & 1.00 & $22(55.0)$ & $18(45.0)$ & 0.50 \\
\hline MAP (mm Hg), mean (SD) & $61(11)$ & $64(9)$ & 0.20 & $57(16)$ & $56(21)$ & 0.85 \\
\hline Heart rate (beats/min), mean (SD) & $94(24)$ & $86(22)$ & 0.15 & $95(32)$ & $77(33)$ & 0.02 \\
\hline $\operatorname{LVEF}(\%)$, median $(\mathrm{Q} 1, \mathrm{Q3})$ & $27.5(20,40)$ & $30(22.5,37.5)$ & 0.76 & $15(10,25)$ & $15(10,25)$ & 0.77 \\
\hline Lactate (mmol/L), median $(\mathrm{Q} 1, \mathrm{Q3})$ & $3.5(1.8,5.7)$ & $3.2(2.0,5.9)$ & 0.92 & $8.5(4.5,11.7)$ & $8.3(3.7,13.4)$ & 0.72 \\
\hline eGFR (mL/min/1.73 m²), mean (SD) & $62(24)$ & $60(21)$ & 0.67 & $63(21)$ & $60(19)$ & 0.61 \\
\hline In-hospital management and outcome & & & & & & \\
\hline 30-day mortality, n (\%) & $11(27.5)$ & $15(37.5)$ & 0.32 & $16(40.0)$ & $31(77.5)$ & $<0.001$ \\
\hline Cause of in-hospital death, $\mathrm{n}(\%)$ & & & & & & \\
\hline Cardiac & $8(20.0)$ & $7(17.5)$ & 0.78 & $7(17.5)$ & $18(45.0)$ & 0.008 \\
\hline Multiorgan failure & $1(2.5)$ & $3(7.5)$ & 1.00 & $6(15.0)$ & $5(12.5)$ & 1.00 \\
\hline Anoxic brain injury & $1(2.5)$ & $2(5.0)$ & 1.00 & $2(5.0)$ & $3(7.5)$ & 1.00 \\
\hline Other, n (\%) & $1(2.5)$ & $2(5.0)$ & 1.00 & $1(2.5)$ & $2(5.0)$ & 1.00 \\
\hline Mechanical ventilation, $n(\%)$ & $24(60.0)$ & $27(67.5)$ & 0.49 & $36(90.0)$ & $35(87.5)$ & 1.00 \\
\hline Vasoactive drug use overall, n (\%) & $36(90.0)$ & $40(100)$ & 0.12 & 39 (97.5) & 39 (97.5) & 1.00 \\
\hline Escalating MCS, n (\%) & $2(5.0)$ & $19(47.5)$ & $<0.001$ & $5(12.5)$ & $12(30.0)$ & 0.10 \\
\hline Use of VA-ECMO & $0(0.0)$ & $0(0.0)$ & 1.00 & $4(10.0)$ & $7(17.5)$ & 0.35 \\
\hline Renal replacement therapy for AKI & $8(20.5)$ & $8(21.0)$ & 0.95 & $19(48.7)$ & $11(28.2)$ & 0.10 \\
\hline Limb ischaemia & $1(2.6)$ & $1(2.5)$ & 1.00 & $4(10.0)$ & $3(7.5)$ & 1.00 \\
\hline
\end{tabular}

AKI, acute kidney injury; BMI, body mass index; COPD, chronic obstructive pulmonary disease; DM, diabetes mellitus; eGFR, estimated glomerular filtration rate; IABP, intra-aortic balloon pump; IHD, ischaemic heart disease; LVEF, left ventricular ejection fraction; MAP, mean arterial pressure; MCS, mechanical circulatory support; MI, myocardial infarction; PAD, peripheral arterial disease; PCl, percutaneous coronary intervention; ROSC, return of spontaneous circulation; STEMI, ST elevation myocardial infarction; VA-ECMO, venoarterial extracorporeal membrane oxygenation.

pre-PCI subgroup analysis. ${ }^{6}$ Other observational studies have compared the effect of Impella application pre-PCI versus post-PCI, and the results favour pre-PCI Impella. ${ }^{78}$
These studies may suffer from confounding by indication, as patients in whom PCI fails to stabilise haemodynamics and receive Impella post-PCI may have a worse outcome 


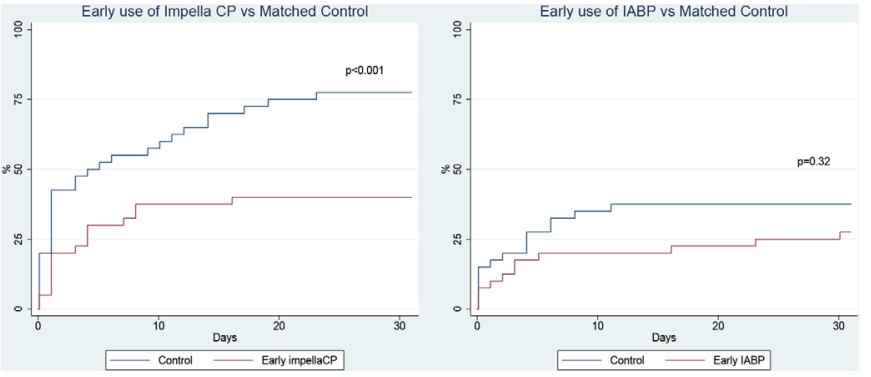

Figure 4 Kaplan-Meier curves of 30-day mortality with early-Impella CP versus propensity score-matched controls and early-IABP versus propensity score-matched controls. Propensity score was calculated based on age, left ventricular ejection fraction, lactate levels, estimated glomerular filtration rate and cardiac arrest before arrival to the cardiac catheterisation laboratory. IABP: intra-aortic balloon pump.

compared with those receiving Impella pre-PCI. Experimental animal studies have demonstrated that deploying an unloading MCS system prior to reperfusion has been demonstrated to reduce infarct size in various species, and that a strategy of Impella CP and norepinephrine improved end-organ perfusion without increasing myocardial workload in a large porcine model of profound cardiogenic shock. ${ }^{18-21}$ In the present study, the improved survival effect of early Impella CP was primarily driven by fewer in-hospital cardiac deaths, supporting the association of improved outcome with early left ventricular unloading. However, no definite conclusions can be drawn from this observational study that at the best should be considered hypothesis generating.

\section{LIMITATIONS}

The clear-cut change in choice of device (primarily IABP from 2010 to 2012, and primarily Impella CP from 2013 to 2017) caused no overlap in time when the two devices were at disposal simultaneously, and given the striking difference in the patient population receiving MCS across the two time periods, we compared early use of IABP and early use of Impella CP to their nearest neighbour control within their respective time periods, instead of comparing early IABP to early Impella CP. In the absence of randomised controlled trials, the propensity score is a method to obtain balance between groups in the search for causal inference and is defined as the probability of receiving treatment given the observed baseline covariates. ${ }^{22}$ One-to-one nearestneighbour matching is superior to the classic regression analysis in reducing bias, especially if covariates are poorly balanced between groups. ${ }^{23}$ Despite propensity matching, the present study carries the risk of confounding by indication, selection bias and bias due to unmatched and unobserved covariates. We chose to exclude patients dying in the catheterisation laboratory before receiving intensive care treatment, which may have underestimated the effect of MCS. However, matched controls in such a setting would have included patients where MCS was abandoned due to futility, which would have led to an overestimation of the MCS effect. We speculate that additional selection bias might have occurred in the early-IABP group, given the lower rate of in-hospital deaths due to multiorgan failure since the IABP does not improve end-organ perfusion or risk of multiorgan dysfunction as demonstrated in an experimental porcine model of moderate acute heart failure and IABP-SHOCK I and II trials. ${ }^{3425}$ The possibility of additional selection bias in the early-Impella CP versus matched control group cannot be excluded as well. Overmatching is a potential limitation as we cannot exclude the possibility of assessment of LVEF/measurement of arterial lactate and creatinine having occurred after initiation of early IABP/Impella CP in some cases. The probability of undermatching is less likely as matching variable strata were narrow and no residual confounding was observed of the continuous variables within their respective strata. Additional overestimation and underestimation of treatment effects could also have occurred, and the influence of a learning curve with the use of Impella CP cannot be excluded. The number of patients who received an early device was limited, and this study does not replace the need for a randomised controlled trial. We do, however, find it encouraging that 30-day mortality improved with earlyImpella CP versus matched control group, and not with early-IABP versus matched control group, using the same matching procedure.

\section{CONCLUSION}

In this historical follow-up study of patients with AMICS undergoing PCI within 24 hours of symptom onset in southeastern Denmark, the use of MCS decreased by $50 \%$ and a shift from IABP to mostly Impella CP occurred following the IABP-SHOCK II trial. Patients receiving MCS after 2012 were younger and presented with more compromised haemodynamics compared with patients receiving MCS prior to 2012. When compared with their respective temporal matched control group, early application of Impella CP was associated with improved survival, while early application of IABP was not.

\section{Author affiliations}

${ }^{1}$ Department of Cardiology, Odense Universitetshospital, Odense, Denmark

${ }^{2}$ Emergency Medicine, Regional Hospital Randers, Randers, Midtjylland, Denmark

${ }^{3}$ Odense Patient data Explorative Network, University of Southern Denmark, Odense, Denmark

${ }^{4}$ Department of Cardiology, Rigshospitalet, Copenhagen, Denmark

${ }^{5}$ Department of Clinical Medicine, University of Copenhagen, Copenhage, Denmark ${ }^{6}$ Department of Anesthesiology and Intensive Care, Odense University Hospital, Odense, Denmark

${ }^{7}$ Department of Cardiothoracic Anaesthesiology, Rigshospitalet, Kobenhavn, Denmark

\section{Twitter Lene Holmvang @LHolmvang}

Contributors Conception and design: OKLH, CH, LOJ, LH, HS, HBR and JEM. Data collection: OKLH, JJ, NLJU, LOJ and LH. Data analysis and interpretation: OKLH, CH, 
HBR and JEM. Drafting of the article: OKLH, CH, HBR and JEM. Critical revision and final approval: all authors.

Funding This work was supported by the Danish Heart Foundation, the Odense University Hospital/Rigshospitalet Research Fund and a research grant from Abiomed.

Competing interests OKLH and NLJU received travel compensation from Abiomed. JEM received research grants and speaker's fee from Abiomed.

Patient consent for publication Not required.

Ethics approval This study was approved by the Danish Patient Safety Authority (case number 3-3013-1133/1) and the Danish Data Protection Agency (file number $16 / 7381$ and 18/23756).

Provenance and peer review Not commissioned; externally peer reviewed.

Data availability statement Data are available upon reasonable request. Deidentified participant data can be made availible upon reasonable request.

Open access This is an open access article distributed in accordance with the Creative Commons Attribution Non Commercial (CC BY-NC 4.0) license, which permits others to distribute, remix, adapt, build upon this work non-commercially, and license their derivative works on different terms, provided the original work is properly cited, appropriate credit is given, any changes made indicated, and the use is non-commercial. See: http://creativecommons.org/licenses/by-nc/4.0/.

ORCID iD

Ole Kristian Lerche Helgestad http://orcid.org/0000-0003-3847-8758

\section{REFERENCES}

1 Helgestad OKL, Josiassen J, Hassager C, et al. Temporal trends in incidence and patient characteristics in cardiogenic shock following acute myocardial infarction from 2010 to 2017: a Danish cohort study. Eur J Heart Fail 2019;21:1370-8.

2 Goldberg RJ, Spencer FA, Gore JM, et al. Thirty-year trends (1975 to 2005) in the magnitude of, management of, and hospital death rates associated with cardiogenic shock in patients with acute myocardial infarction: a population-based perspective. Circulation 2009;119:1211-9.

3 Thiele H, Zeymer U, Neumann F-J, et al. Intraaortic balloon support for myocardial infarction with cardiogenic shock. N Engl J Med 2012;367:1287-96.

4 Shah M, Patnaik S, Patel B, et al. Trends in mechanical circulatory support use and hospital mortality among patients with acute myocardial infarction and non-infarction related cardiogenic shock in the United States. Clin Res Cardiol 2018;107:287-303.

5 Strom JB, Zhao Y, Shen C, et al. National trends, predictors of use, and in-hospital outcomes in mechanical circulatory support for cardiogenic shock. Eurolntervention 2018;13:2152-9.

6 Schrage B, Ibrahim K, Loehn T, et al. Impella support for acute myocardial infarction complicated by cardiogenic shock. Circulation 2019;139:1249-58.

7 Jensen PB, Kann SH, Veien KT, et al. Single-centre experience with the Impella CP, 5.0 and RP in 109 consecutive patients with profound cardiogenic shock. Eur Heart J Acute Cardiovasc Care 2018;7:53-61.

8 O'Neill WW, Grines C, Schreiber T, et al. Analysis of outcomes for 15,259 us patients with acute myocardial infarction cardiogenic shock (AMICS) supported with the Impella device. Am Heart J 2018;202:33-8.

9 Huang C-C, Hsu J-C, Wu Y-W, et al. Implementation of extracorporeal membrane oxygenation before primary percutaneous coronary intervention may improve the survival of patients with STsegment elevation myocardial infarction and refractory cardiogenic shock. Int J Cardiol 2018;269:45-50.

10 Ouweneel DM, Eriksen E, Sjauw KD, et al. Percutaneous Mechanical Circulatory Support Versus Intra-Aortic Balloon Pump in Cardiogenic Shock After Acute Myocardial Infarction. J Am Coll Cardiol 2017;69:278-87.

11 Thiele H, Ohman EM, Desch S, et al. Management of cardiogenic shock. Eur Heart J 2015;36:1223-30.

12 Kapur NK, Davila CD. Timing, timing, timing: the emerging concept of the 'door to support' time for cardiogenic shock. Eur Heart $J$ 2017;38:3532-4.

13 Levey AS, Stevens LA, Schmid $\mathrm{CH}$, et al. A new equation to estimate glomerular filtration rate. Ann Intern Med 2009;150:604-12.

14 Udesen NJ, Møller JE, Lindholm MG, et al. Rationale and design of danger shock: Danish-German cardiogenic shock trial. Am Heart J 2019;214:60-8.

15 Rathod KS, Koganti S, lqbal MB, et al. Contemporary trends in cardiogenic shock: incidence, intra-aortic balloon pump utilisation and outcomes from the London heart attack group. Eur Heart $J$ Acute Cardiovasc Care 2018;7:16-27.

16 Becher PM, Schrage B, Sinning CR, et al. Venoarterial extracorporeal membrane oxygenation for cardiopulmonary support. Circulation 2018;138:2298-300.

17 Alushi B, Douedari A, Froehlig G, et al. Impella versus IABP in acute myocardial infarction complicated by cardiogenic shock. Open Heart 2019;6:e000987

18 Meyns B, Stolinski J, Leunens V, et al. Left ventricular support by catheter-mounted axial flow pump reduces infarct size. J Am Coll Cardiol 2003;41:1087-95.

19 Kapur NK, Paruchuri V, Urbano-Morales JA, et al. Mechanically unloading the left ventricle before coronary reperfusion reduces left ventricular wall stress and myocardial infarct size. Circulation 2013;128:328-36.

20 Kapur NK, Qiao X, Paruchuri V, et al. Mechanical pre-conditioning with acute circulatory support before reperfusion limits infarct size in acute myocardial infarction. JACC Heart Fail 2015;3:873-82.

21 Møller-Helgestad OK, Hyldebrandt JA, Banke A, et al. Impella CP or VA-ECMO in profound cardiogenic shock: left ventricular unloading and organ perfusion in a large animal model. Eurolntervention 2019;14:e1585-92.

22 ROSENBAUM PR, RUBIN DB. The central role of the propensity score in observational studies for causal effects. Biometrika 1983;70:41-55

23 Rubin DB. Using multivariate matched sampling and regression adjustment to control bias in observational studies. J Am Stat Assoc 1979;74:318-28

24 Møller-Helgestad OK, Poulsen CB, Christiansen EH, et al. Support with intra-aortic balloon pump vs. Impella $2.5 \AA$ and blood flow to the heart, brain and kidneys - an experimental porcine model of ischaemic heart failure. Int J Cardiol 2015;178:153-8.

25 Prondzinsky R, Lemm H, Swyter M, et al. Intra-Aortic balloon counterpulsation in patients with acute myocardial infarction complicated by cardiogenic shock: the prospective, randomized IABP shock trial for attenuation of multiorgan dysfunction syndrome. Crit Care Med 2010;38:152-60. 